\title{
béton compacté au rouleau
}

\author{
roller compacted concrete
}

\author{
par André GOUBET* \\ Ingénieur Général des Ponts et Chaussées \\ Président du Comité Technique Permanent des Barrages
}

\section{Résumé}

Cet article rappelle la façon dont a pris naissance et s'est développée la technique du Béton Compacté au Rouleau (B.C.R.) et analyse tout particulièrement les divergences constatées jusqu'à présent sur de nombreux points dans ses modalités de mise en œuvre dans les barrages (agrégats, liants, épaisseur des couches, traitement des joints de reprise, obtention de l'imperméabilité). Cette diversité prouve la nécessité de réflexions complémentaires approfondies susceptibles de déboucher sur des barrages d'une conception sensiblement différente de celle des barrages-poids classiques.

\section{Abstract}

This article recalls the beginnings and the way the technique of Roller Compacted Concrete was developed. It especially analyses the differences that have been noted up till now on many points concerning its application in dam works (aggregates, pastes, lifts, lift lines, waterproofing). This diversity proves that it is necessary to reflect seriously about complementary methods in order to arrive at a conception of a dam that is hardly different from a conventional gravity dam.

\footnotetext{
- Ministère de l'industrie des P.-et-T, et du Tourisme. Direction du gaz, de l'électricité et du charbon, 120, rue du Cherche-Midi, 75006 Paris.
} 


\section{INTRODUCTION}

L'expression « Béton Compacté au Rouleau » et le sigle correspondant « B.C.R. » se sont imposés dans la langue française pour désigner un ensemble de produits ou de techniques désignés par des expressions variées dans la littérature anglaise : «Rolled concrete in dam * (R.C.D.) utilisé par les Japonais, « Dry lean concrete» (D.L.C.), «Roller compacted concrete» (R.C.C.), «Rollcrete », utilisés par les auteurs de langue anglaise avec des significations parfois divergentes (1).

Nous allons tenter successivement de définir le B.C.R.; d'exposer les différentes démarches ou recherches, parfois contradictoires, qui se sont développées entre 1960 et 1980 pour aboutir à un premier ensemble de réalisations au début des années 80 ; de préciser, à partir de ces réalisations, les points communs et les variantes importantes; puis enfin d'esquisser quelques perspectives d'avenir.

\section{DÉFINITION}

Le B.C.R. est un béton essentiellement mis en cuure à l'aide des matériels classiques de terrassement, à l'exclusion des matériels spécifiques aux chantiers de barrage en béton classique: blondins, grues tours, aiguilles de vibration, souvent couteux d'installation et difficiles à amortir sur plusieurs chantiers. Le béton (2) est amené en vrac, à son point d'utilisation par camions ou par tapis, étendu en couches minces par bulldozer puis compacté au rouleau lourd, en général vibrant. Ces éléments, communs à tous les barrages en B.C.R., induisent un certain nombre d'autres caractères identiques mentionnés plus loin.

\section{APPARITION PROGRESSIVE DU B.C.R.}

Avant-guerre, et même dans les premières années qui lont suivie, une proportion importante des barrages était réalisée en béton. Les progrès enregistrés en matière d'engins de terrassement et de mécanique des sols ont provoqué une régression importante des ouvrages en béton (3). Aiguillonnés par cette concurrence, à partir des années 1960 , plusieurs spécialistes du béton s'efforcèrent d'imaginer des procédés qui permettraient des économies substantielles.

Une première idée est de s'affranchir des plots coffrés : le barrage d'Alpe Gera (4), réalisé en 1964 en Italie, est parfois considéré comme l'ancêtre des barrages en B.C.R... à ceci près que l'on n'a pas utilisé de rouleau! Mais les autres éléments du B.C.R. sont déjà rassemblés : amenée par dumper, régalage au bulldozer sur

\footnotetext{
(1) Dans le numéro de janvier 1986 de Water Power and Dam Construction, le terme «rollcrete est utilisé par un auteur sud. africain pour désigner l'ensemble des B.C.R. tandis que les Américains réservent plutôt cette expression aux bétons roulés de type grave-ciment.
}

(2) Je continue à utiliser ce terme dont nous verrons plus loin qu'il peut prêter à confusion. toute la surface du barrage. Les vibrateurs à aiguilles restent toutefois utilisés et les joints de contraction sont découpés dans le béton fraîchement répandu. Quatre ans plus tard le même constructeur récidive avec le barrage de Quourra della Miniera, toujours en Italie... et la lignée semble s'éteindre (au moins dans ce pays).

A la fin des années 1960 Hydro Quebec propose de distinguer nettement dans les barrages-poids le parement amont chargé d'assurer l'étanchéité, riche en liant, muni de waterstops et réalisé dans une première phase à l'aide de coffrages glissants, le parement aval constitué par des blocs préfabriqués et un coeur intermédiaire constitué par un béton très pauvre $(120 \mathrm{~kg}$ de liant dont moitié de cendres volantes ou de pouzzolane) mis en place par couches minces continues sur toute l'étendue du barrage, sans aucune précaution particulière entre couches.

Contrairement à Alpe Gera, il n'y a plus aucune vibration de la partie centrale mais on ne parle pas encore de rouleau (5). Un prototype est réalisé à l'aménagement de Manicouagan I ( 2 barrages latéraux de $18 \mathrm{~m}$ de haut).

La première allusion à l'emploi du rouleau pour compacter le béton de barrages-poids semble avoir été présentée par PATON (Grande-Bretagne) au congrès de la C.I.G.B. de Montréal en 1970. PATON se référait à son expérience d'ingénieur routier ayant souvent mis en œuvre, pour réaliser des chaussées (et non des sous-couches), un béton pauvre et sec (dry lean concrete) fortement compacté au rouleau vibrant et acquérant une densité élevée. Mais il signale lui-même le manque de connaissance sur la perméabilité des contacts entre couches.

Par ailleurs, dès 1960, le noyau étanche du batardeau du barrage de Shimen à Formose, est réalisé avec un sol amélioré au ciment, le matériau disponible localement étant trop humide pour permettre une réalisation classique dans les délais souhaités. Mais cette réalisation, demeurée isolée, s'inspire davantage du solciment précédemment utilisé pour les revêtements amont des digues en terre que d'un souci de minorer le coût des barrages-poids en béton.

De son côté, en 1970, aux U.S.A., RAPHAËL propose comme un optimum entre les barrages en terre et les barrages-poids classiques, un barrage à profil épais constitué par des matériaux bruts d'extraction et enrichis au ciment.

On constate donc dès 1970 l'affirmation de deux tendances, lune relative à un béton roulé à agrégats classiques, l'autre à un béton plus proche des "gravesciments $x$.

(3) Entre 1945 et 1964, 40\% des grands barrages construits dans le monde étaient en béton (dont $60 \%$ de barrages-poids); depuis 1968 , cette proportion est tombée aux environs de $17 \%$.

(4) $178 \mathrm{~m}$ de haut - $1730000 \mathrm{~m}^{3}$ de béton, situé à une altitude de $2100 \mathrm{~m}$.

(5) Il est vrail que le réglage en couche mince par les bulldozers introduit déjà un compactage efficace. 
Cette dernière approche va donner lieu à une réalisation spectaculaire entre 1974 et 1979 à Tarbela où 2,3 millions de $\mathrm{m}^{3}$ de rochers arrachés par l'érosion sont remplacés par du « rollcrete ». Toutefois, ce travail n'intéresse pas le barrage proprement dit.

Mais pendant les années 70 , on en demeure essentiellement au stade des réflexions et d'essais plus ou moins importants aux U.S.A., en Grande-Bretagne et surtout au Japon.

C'est dans ce pays que les études sont menées le plus systématiquement car une commission spéciale est officiellement mise en place par le Gouvernement en 1974. Une «technique japonaise est ainsi officiellement établie, et baptisée en anglais R.C.D. (rolled compacted dam), technique qui a légèrement évolué au cours des années mais qui se caractérise essentiellement par la réalisation d'un béton de très bonne qualité utilisé pour réaliser des barrages-poids de profil classique à plots indépendants. L'économie de prix par rapport aux barrages-poids classiques n'est que de 10 à $15 \%$.

Après un essai au cours duquel $13000 \mathrm{~m}^{3}$ sont coulés pour constituer la protection de la vallée à l'aval du barrage de Shin-Nakano (1980), le Japon construit les barrages de Shimajigawa ( $89 \mathrm{~m}$ de haut, 1980), Ohkawa (75 m, 1980) (1), Tamagawa (103 m, 1986, 1100000 (2)) et Pirika (34 $\mathrm{m}$, en construction en 1986).

Aux U.S.A. ou en Grande-Bretagne, pour supplanter les barrages en remblais, on recherche des gains plus substantiels de prix par rapport aux barrages-poids classiques. Différents ingénieurs élaborent, toujours au cours des années 1970 , des projets de conception variée qui devaient aboutir à des réalisations dans le début des années 80 .

En Grande-Bretagne, le barrage de Holbeam Wood est achevé en 1982. De caractéristiques modestes, ce barrage écrêteur de crue, qui ne devrait être rempli que quelques jours par siècle, est constitué par un profil poids encadré à l'amont et à l'aval de recharges en terre jugées plus esthétiques. Par ailleurs, le barrage de Milton Brook, qui a fait l'objet de longues études, semble avoir été construit vers 1985 .

Aux États-Unis, début 1986, 4 barrages importants sont terminés : Willow Creek (52 m, 1982, 331000), Winchester (21 m, 1984, 24500), Middle Fork (38 m, $1984,42000)$ et Galesville $(51 \mathrm{~m}, 1985,17000)$ et 4 en construction : Upper Stillwater, ( $82 \mathrm{~m}, 1987$. $1070000)$, Monksville (46 m, 1986, 221000), Grindstone Canyon (42 m, 1986, 87500) et Elk Creek (76 m, 1988, 796000).

A partir de 1980 les conceptions américaines commencent à se répandre à travers le monde.

(1) En fait seule est réalisée en B.C.R. une couche de fondation de $25 \mathrm{~m}$ d'épaisseur environ qui s'étend à la fois sous le barrage et sous le bassin de dissipation.

(2) La valeur éventuellernent mentionnée après la date de mise en service est le volume de B.C.R.
C'est ainsi que l'on peut par ailleurs signaler des réalisations récentes en Australie (Kidston, 40 m, 1984), au Brésil (Saco de Nova Olinda, $57 \mathrm{~m}$, dont le bétonnage s'est terminé en 1986, et Varzea Grande, 33 m, qui devait être terminé pour la fin de 1986), en Afrique du Sud (Mistkroal Weir, $30 \mathrm{~m}, 60000 \mathrm{~m}^{3}$ et Zaaihoek, $50 \mathrm{~m}, 120000 \mathrm{~m}^{3}$ étaient en construction en 1985), en Espagne (Castilblanco de los Arroyos, $25 \mathrm{~m}$, $18000 \mathrm{~m}^{3}$.

De nombreux projets sont par ailleurs en cours ou sur le point de démarrer, dont, parmi les plus importants, Trigomil, au Mexique, qui avec ses $99 \mathrm{~m}$ de haut battrait le record d'Amérique (début de bétonnage à l'automne 86) et Urugua-i, en Argentine $(77 \mathrm{~m}$ de haut, début de bétonnage en juillet 1987).

En France le barrage des Olivettes, en voie d'achèvement, constitue la première réalisation importante. Avec ses $36 \mathrm{~m}$ et ses $80000 \mathrm{~m}^{3}$ de béton il occupe une place honorable parmi les diverses réalisations mondiales. Diverses solutions originales ont été mises en ceuvre lors de sa réalisation et nous en reparlerons donc ultérieurement. Il avait été précédé par la construction d'une petite digue à Saint-Martin-de-Londres et du batardeau du barrage de Pont-de-Veyrière.

Notons dès à présent que presque tous ces ouvrages appartiennent à la catégorie «béton à agrégats classiques $n$.

Cet aperçu ne serait pas complet si l'on ne signalait pas l'utilisation du B.C.R. dans les réalisations pour lesquelles les qualités exigées peuvent être très différentes de celles requises pour les barrages-poids : au noyau du batardeau du barrage de Shimen, cité ci-dessus, on peut associer ceux des batardeaux des barrages de Karun (Iran) et d'Al Massira (Maroc). Le batardeau du barrage de Veyrière (France) a été réalisé uniquement en B.C.R., à titre de test. Plusieurs barrages en terre aux U.S.A. ont été renforcés par un revêtement aval en B.C.R., ce qui améliore notamment très sensiblement la résistance aux submersions éventuelles. Par ailleurs, le B.C.R. a été utilisé sur certains sites de barrages-poids pour remplacer ou compléter un rocher de qualité médiocre dans les fondations ou à l'aval, sans pour autant constituer le barrage proprement dit : ce fut le cas non seulement pour le barrage de ShinNakano (Japon) cité ci-dessus, mais aussi pour le Saddam dam (Irak) où un million de mètres cubes de B.C.R. a été mis en place.

Enfin des aires de stockage, des parkings et même des routes à fort trafic ont été réalisés en B.C.R.

\section{CARACTÈRES COMMUNS AUX DIVERSES RÉALISATIONS EN B.C.R.}

Les B.C.R. sont essentiellement des bétons étalés en couches minces (en pratique toujours par des bulldozers) et compactés plus énergiquement par des rouleaux vibrants que par les aiguilles de vibration classiques. Par ailleurs, le passage des rouleaux ne peut s'effectuer que sur un produit très sec. Ces deux éléments permettent d'obtenir un béton qui, à qualité et 
quantité égales d'agrégats et de liants a de meilleures caractéristiques mécaniques, est plus étanche dans la masse et présente moins de retrait.

D'un béton à l'autre, on peut utiliser des agrégats et des liants très divers et obtenir, comme d'ailleurs pour le béton classique, des résultats également très divers.

Par exemple le B.C.R. permet de réaliser des chaussées routières sans joints transversaux (faible retrait) ou de remplacer du béton classique armé pour des aires de stockages.

Par contre, il présente un inconvénient grave dès que l'on veut réaliser un ouvrage de plus de 50 à $100 \mathrm{~cm}$ d'épaisseur : l'étanchéité du contact entre deux couches successives est médiocre et la perméabilité moyenne d'un massif important, tel un barrage, laisse à désirer.

Les causes de cette perméabilité, et donc la manière de la réduire, sont mal élucidées : on met le plus souvent en avant le fait que la couche inférieure a déjà partiellement fait prise avant d'être recouverte. Mais il semble que la différence de granulométrie entre la partie supérieure d'une couche, dans laquelle la laitance a tendance à remonter, et la partie inférieure de la couche suivante joue également un rôle. La plus ou moins grande richesse en liant aurait aussi des conséquences sensibles.

On considère parfois le B.C.R. comme un produit intermédiaire en béton classique et terre. Une telle analyse est ambiguë et peut prêter à confusion. $\mathrm{Ce}$ n'est pas parce que le béton est vibré au rouleau plutôt qu'à l'aiguille, transporté au camion benne plutôt que manutentionné au blondin et étalé au bulldozer plutôt qu'à la main, qu'il cesse d'être du béton, c'est-à-dire un matériau doué d'une cohésion importante et constituant des barrages rigides.

Par contre le coût du mètre cube de B.C.R. mis en œuvre dans un barrage-poids peut être 2 à 3 fois infêrieur (1) à celui du béton classique et la solution optimale convenant à chaque barrage pourrait s'écarter sensiblement de celle correspondant au volume minimal de béton; elle peut correspondre à un ouvrage dont le volume est intermédiaire entre ceux d'un barrage en terre et en béton classique et dont les dispositions constructives sont assez éloignées de celles d'un tel barrage en béton.

Or, on constate qu'actuellement tous les barrages réalisés s'inspirent très directement des solutions adaptées antérieurement pour les barrages-poids et retiennent des profils très voisins des profils classiques.

Plusieurs éléments sont communs à la totalité ou à la quasi-totalité des réalisations actuelles :

1. L'intérieur du barrage doit avoir un dessin aussi simple que possible, car tous les obstacles aux déplacements des engins de terrassement (galeries, conduites diverses) entraînent des sujétions importantes.

(1) Ce rapport varie évidemment avec les quantités mises en place et s'entend pour un B.C.R. dont les contacts entre couches n'ont pas fait l'objet d'un traitement particulier.
2. Les liants contiennent toujours une proportion élevée de cendres volantes ou de pouzzolane (2). On a toujours cherché à réduire l'élévation de température provoquée par la prise du béton des barrages-poids et l'emploi de cendres volantes est bien antérieur au B.C.R. Toutefois, dans les barrages en B.C.R. le recours aux liants à prise lente est encore plus indiqué car :

- on s'efforce souvent de réaliser des barrages monolithiques d'une rive à l'autre:

- on souhaite souvent que la couche inférieure ait à peine amorcé sa prise avant mise en place de la couche suivante.

Bien entendu, dans certaines régions (Moyen Orient) on ne pourrait trouver de cendres volantes à un prix acceptable et force serait de faire sans.

3. Les cadences de bétonnage sont presque toujours très élevées et nécessitent pratiquement toujours des stations de bétonnage en continu dérivées des installations routières. Ces cadences élevées, analogues à celles observées dans les barrages en remblai, constituent d'ailleurs l'un des avantages économiques importants du B.C.R. C'est ainsi que l'on a atteint des productions journalières de $4460 \mathrm{~m}^{3}$ à Willow Creek, près de 5700 à Galesville, 5800 à Tamagawa, et que l'on prévoyait 7650 à Upper Stillwater et 9550 à Elk Creek.

4. On annonce souvent des économies spectaculaires par rapport aux réalisations classiques. Mais peut-être faudrait-il attendre, pour se faire une opinion définitive en la matière, que soient soldées certaines réclamations d'entreprises, également spectaculaires.

\section{DIVERGENCES ENTRE LES DIVERSES RÉALISATIONS EN B.C.R.}

Dans le rapport général de la question 57 du congrès de la C.I.G.B. à Lausanne (1985) le Rapporteur passait en revue 10 points à propos desquels de larges divergences étaient constatées entre les réalisations existantes.

Cela ne doit pas surprendre; même en ce qui concerne les barrages-poids classiques, on note des différences importantes par exemple quant aux dimensions, en surface ou en épaisseur des plots et des levées successives.

Ces divergences peuvent aussi résulter des objectifs des barrages, de leur situation géographique (climat plus ou moins froid), de leur taille (et donc du rapport volume du noyau central/surface des parements), ou tout simplement de la hardiesse des projeteurs plus ou moins ambitieux dans l'utilisation d'un procédé de construction nouveau. Mais elles sont imputables pour l'essentiel au niveau d'étanchéité global recherché pour le barrage et au moyen d'assurer cette étanchéité, soit par un élément amont, soit par le B.C.R. lui-même.

(2) En France, pour les barrages de Saint-Martin de Londres et des Olivettes, on utilise même un mélange sans clinker, composé uniquement de laitier de haut fourneau et de cendres volantes de Gardanne. 
Présentons une revue rapide de l'éventail des solutions adoptées :

- Réalisation de l'étanchéité : nous avons vu que la faiblesse du B.C.R. est sa perméabilité au contact de deux couches successives, perméabilité qui semble d'ailleurs s'atténuer rapidement si les eaux de la retenue sont chargées. Dans certains cas, rares il est vrai, cette perméabilité est acceptable moyennant éventuellement quelques précautions dans la formulation du béton (plus riche en liant) ou sa mise en ceuvre : barrages de lutte contre les crues (Willow Creek, Holbeam Wood), batardeau du Pont de Veyrière, noyau des batardeaux de Shimen, Karun (50 m, 1971), Al Massira (20 m, 1978).

Dans la plupart des cas, il faut réduire la perméabilité soit en créant une étanchéité amont (béton classique, membrane de P.V.C., enduit plastique) soit en améliorant l'étanchéité dans la masse en étalant une couche de quelques centimètres de mortier sur une plus ou moins grande surface sous chaque couche de B.C.R. ; ces dispositions sont évidemment couteuses.

De cette multiplicité de solutions possibles découle une bonne partie des options très diverses constatées par ailleurs; par exemple une excellente et couteuse étanchéité amont permet ensuite d'adopter un B.C.R. moins riche en liant, donc moins susceptible de se fissurer, ...

Notons que l'on a constaté que certains barrages en B.C.R., qui présentaient initialement quelques fuites, ont vu ensuite leur étanchéité évoluer très favorablement.

- Teneur en liant : les cendres volantes, presque toujours présentes, jouent un rôle dans le mécanisme de prise du liant (rôle éventuellement variable d'ailleurs avec l'origine du charbon), mais peuvent également être utilisées pour augmenter la teneur en fine d'agrégats qui en seraient naturellement dépourvus. Ceci peut expliquer les pourcentages très différents constatés d'une réalisation à l'autre (20 à $80 \%)$; une autre cause de variation, rarement signalée de façon explicite, est le rapport des cout du ciment et des cendres volantes.

Mais par ailleurs, les teneurs en liant sont également très différentes, certains barrages ayant été réalisés avec des B.C.R. de richesse différente au centre et pour les parements.

On peut comparer les valeurs suivantes $\left(e n \mathrm{~kg} / \mathrm{m}^{3}\right)$ :

\begin{tabular}{|c|c|c|c|}
\hline Shimajigawa & ciment 84 & Cendres volantes & 36 (cceur) \\
\hline Willow Creek & $\begin{array}{r}175 \\
80 \\
104\end{array}$ & & $\begin{array}{c}0 \text { (amont) } \\
19 \text { (cœeur) } \\
47 \text { (aval) }\end{array}$ \\
\hline Upper Stillwater & $\begin{array}{r}187 \\
92 \\
77\end{array}$ & & $\begin{array}{l}80 \text { (déversoir) } \\
205 \text { (amont) } \\
170 \text { (cœeur) }\end{array}$ \\
\hline
\end{tabular}

Par ailleurs, à Monksville, on n'utilise pas de cendres volantes, les agrégats non lavés étant jugés assez riches en fines. Middle Fork n'utilise également que du ciment.
- Nature et dimension des agrégats : les agrégats sont parfois constitués par du tout venant de carrière (Tarbela) ou par les matériaux extraits des fondations du barrage, non lavés et simplement écrêtés (Galesville) ou enrichis en éléments moyens (Monksville). Mais souvent on retient des agrégats lavés respectant un fuseau granulométrique strict.

Les différences sont également considérables en ce qui concerne la taille de l'agrégat maximum : tous les experts sont d'accord pour admettre que l'introduction de gros éléments permet de diminuer la teneur en liant et donc, indépendamment des coûts, de diminuer le dégagement de chaleur. Mais, par ailleurs, de trop gros agrégats pourraient être cause d'une ségrégation que tous veulent éviter; toutefois, la taille maximum varie de $40 \mathrm{~mm}$ à Upper Stillwater à $150 \mathrm{~mm}$ à Tamagawa ; la valeur la plus communément admise semblant être de l'ordre de $70 \mathrm{~mm}$. En fait, il semble que la lutte contre la ségrégation nécessite essentiellement quelques précautions simples lors du chargement et du déchargement du béton dans les dumpers ainsi que lors du régalage. Par ailleurs, je n'ai jamais vu d'étude sur les inconvénients d'une certaine ségrégation dans le cceur des barrages-poids.

La diversité de la taille maximum d'un projet à l'autre peut également être liée à la diversité des épaisseurs de couches.

- Épaisseur des couches : ici aussi la diversité est grande : de 30 à $100 \mathrm{~cm}$, chaque expert invoquant, pour limiter l'épaisseur, la nécessité de compacter efficacement la partie inférieure de chaque couche.

D'autres motifs me paraissent peser d'un poids au moins équivalent et nécessitent quelques explications détaillées.

Pour éviter un début de prise trop important de la couche supérieure, certains projets prévoient des délais de mise en place de 7 à 8 heures entre 2 couches successives, soit 3 à 4 couches par jour sur toute la surface du barrage. Ceci conduit à travailler 7 jours par semaine et $20 \mathrm{~h}$ par jour et à réaliser $95 \%$ du volume total d'un barrage de $51 \mathrm{~m}$ en 7 semaines (Galesville) ou à mettre en place le R.C.C. d'un barrage de $38 \mathrm{~m}$ en 5 semaines (Middle Fork). Une épaisseur de couche supérieure à $30 \mathrm{~cm}$, associée au choix d'un délai de recouvrement court, imposerait des installations de chantiers manifestement déraisonnables.

A l'opposé les Japonais n'admettent pas le point faible que constitue le contact entre couche : chaque couche est laissée à l'air libre pendant plusieurs jours (2 en général) pendant lesquels elle subit des soins attentifs (arrosage), la couche suivante étant mise en place après lavage soigné et répandage d'une couche de mortier de liaison. On travaille par tiers de barrage ce qui permet un rythme de bétonnage continu, et l'on s'efforce évidemment de mettre en place des couches aussi épaisses que possible pour limiter l'ensemble des tâches liées à chaque reprise. Mais, ces couches épaisses (100 cm prévus pour la moitié supérieure à Tamagawa) sont constituées par 3 (ou parfois 4) souscouches étalées successivement au bulldozer, or on constate que le régalage au bulldozer introduit en fait un compactage important. 
Toutes les premières réalisations aux U.S.A. ont été faites avec des épaisseurs de l'ordre de $30 \mathrm{~cm}$ (1 pied) mais il est prévu 2 pieds pour le barrage d'Elk Creek :

- Parement amont : Les réalisations divergent très largement selon que le parement amont, toujours vertical, doit seulement jouer un rôle mécanique (maintenir le B.C.R. lors du compactage) ou assurer en outre l'étanchéité du barrage (ce choix étant bien entendu lié à celui retenu pour l'étanchéité entre couches de B.C.R.) ; quelques exemples : à Manicouagan on avait réalisé avant mise en place du B.C.R. une paroi amont autostable, en béton classique riche, assurant l'étanchéité. Au Japon, une zone de 3 mètres environ est réalisée de façon classique avec du béton vibré à l'aiguille, elle est montée en même temps que le B.C.R. et l'on s'efforce de solidariser les deux bétons le mieux possible. Au barrage de Winchester le parement amont est constitué de panneaux préfabriqués incorporant une couche de P.V.C. de $1,7 \mathrm{~mm}$ (ce qui impose évidemment de raccorder les bords de cette membrane imperméable d'un panneau à l'autre).

On trouve aussi des éléments préfabriqués autostables (donc assez épais) à Willow Creek ou beaucoup plus minces (et se comportant comme les panneaux des ouvrages en terre armée) à Broom Raubenheimer (petit barrage expérimental réalisé en Afrique du Sud). Enfin, on peut réaliser des murets superposés de forme variable en béton riche, réalisés avec des extrudeuses dérivées des machines utilisées pour construire les séparateurs autoroutiers, ce qui implique évidemment l'absence de joints transversaux (dans ces 2 derniers cas le parement amont n'assure évidemment pas l'étanchéité). Enfin l'étanchéité peut être constituée (ou améliorée) par un enduit plastique appliqué après bétonnage sur le parement amont (Galesville).

- Parement aval : il peut être en B.C.R. ou en béton classique avec plusieurs variantes dans chaque cas. Si le B.C.R. n'est pas coffré (Willow Creek, Galesville, Monkesville) le fruit aval est un peu supérieur à ce qu'exige la stabilité du barrage; le B.C.R. brut présente une apparence inesthétique qui peut être aisément corrigée en tassant légèrement le matériau en surface; mais, même dans ce cas, les qualités mécaniques en surface laissent à désirer et il faut craindre l'effet du gel et la disparition à terme d'une certaine épaisseur de béton.

Généralement le barrage comporte un parement aval de béton ordinaire, épais et mis en place de façon classique (au Japon), ou constitué par des éléments préfabriqués ou par des profils extrudés d'une rive à lautre (Upper Stillwater aux États-Unis).

- Joints transversaux : les Japonais conservent intacts les usages en vigueur pour les barrages-poids classiques: la zone amont en béton vibré comporte tous les $15 \mathrm{~m}$ environ des joints transversaux équipés de waterstop et d'un drain vertical; dans le prolongement, le B.C.R. est scié juste après sa mise en place par une lame vibrante de manière à reconstituer des plots indépendants.

A l'opposé les Américains réalisent généralement des structures monoblocs d'une rive à l'autre après avoir vérifié, à l'aide de modèle mathématique, que les contraintes thermiques n'étaient pas susceptibles de provoquer des tensions excessives. Ce résultat peut éventuellement n'être obtenu que grâce à des précautions particulières : à Monksville ( $46 \mathrm{~m}$ de haut) les $230000 \mathrm{~m}^{3}$ devaient être mis en place du 25 mars au 9 juillet (entre la fin de période de gel et le début des grosses chaleurs) en utilisant des agrégats préparés avant l'hiver et donc à basse température. Notons que pour aucune des réalisations américaines on ne semble avoir pris en considération les possibilités de fissures imputables aux tassements différentiels de la fondation; peut-être n'étaient-elles pas à craindre. Mais à Galesville, 4 fissures sont apparues en septembre 85 , un mois après la fin des travaux; la plus importante, de l'ordre de $0,5 \mathrm{~cm}$, sans être traversante, intéressait à la fois les parements amont et aval (elle ne mettait toutefois pas en cause la stabilité de l'ouvrage).

Un tel mécompte justifie sans doute les solutions intermédiaires retenues sur plusieurs barrages (Manicouagan, les Olivettes) : la paroi amont, en béton classique, comporte de place en place des joints verticaux équipés de waterstops, le B.C.R. étant lui continu. On peut espérer que les fissures éventuelles se produiront au droit de ces joints, constitueront naturellement des plots et n'auront aucune conséquence néfaste.

- Drainage : la plupart des barrages sont drainés de façon classique à l'aide de drains verticaux débouchant dans une galerie horizontale. Ces drains sont parfois des tuyaux mis en place au moment de la construction du parement amont (Japon) mais ils sont la plupart du temps forés a posteriori dans le B.C.R. Toutefois, le barrage de Monksville n'est drainé que dans sa partie déversante : l'épaisseur supplémentaire du profil résultant de l'absence de coffrage permet d'assurer la stabilité en présence d'eau; une seule précaution est prise : la mise en place d'agrégats non cimentés à peu de distance du parement aval permet d'intercepter les fuites dont le gel pourrait dégrader ce parement.

\section{L'AVENIR}

En présence de réalisations aussi diverses, comment envisager l'avenir? La lecture des ceuvres des prix Nobel, et les erreurs de pronostics manifestes dont certains furent victimes, ne peuvent qu'inciter à la plus grande prudence.

Mais on peut observer que les premiers temples de pierre d'Égypte ou de Grèce n'étaient que la copie des ouvrages en bois antérieurs, que les premières églises de béton n'étaient guère que le moulage des bâtiments de pierre. L'homme a mis des siècles pour inventer la voûte, quelques dizaines d'années pour utiliser le voile mince. Seul lavenir dira si le B.C.R. permettra une mutation de cette nature et conduira à des ouvrages fondamentalement différents des barrages mentionnés ci-dessus qui, au-delà de leurs diversités, s'inspirent tous très directement du barrage-poids.

Ne pourrait-on envisager des barrages en B.C.R. à parement amont incliné et revêtus d'une étanchéité analogue à celle des barrages en enrochement : la perméabilité du corps de l'ouvrage, en béton de qualité réduite, serait alors plutôt bénéfique? 
Ne pourrait-on étudier des barrages voûte épais, en B.C.R. de bonne qualité, la compression permettant de limiter la tendance à la fissuration du béton?

Certains supporters enthousiastes n'hésitent pas à prévoir que $60 \%$ des futurs barrages seront réalisés en B.C.R. Partant des 1 ou $2 \%$ actuels, la route est longue et l'objectif peut paraître irréaliste si l'on songe que louvrage en B.C.R. ne peut s'envisager que sur une fondation "rigide». Mais il me parait évident qu'un développement aussi spectaculaire ne sera atteint qu'en ajoutant quelques grammes de matière grise à chaque mètre cube de béton. Comme le disait un ingénieur du Bureau of Reclamation americain atout ouvrage en B.C.R. réalisé dans les 10 prochaines années apportera vraisemblablement une innovation et constituera une approche nouvelle».

Je ne me permettrais qu'un conseil aux projeteurs qui envisagent leur première réalisation : lisez le plus possible, visitez le plus possible, essayez de comprendre, et surtout n'imitez pas mais concevez un ouvrage adapté à son objet, au site, aux agrégats et aux liants disponibles, aux moyens des entreprises... et aux habitudes sociales.

Ou pour reprendre une conclusion plus lapidaire de Dunstan $\propto$ let's roll! », «allons-y, roulons! ». 